\title{
Preliminary Analysis of Force-Torque Measurements for Robot-Assisted Fracture Surgery
}

\author{
Ioannis Georgilas ${ }^{1 *}$, Giulio Dagnino ${ }^{1}$, Payam Tarassoli ${ }^{2}$, Roger Atkins ${ }^{2}$, and Sanja Dogramadzi ${ }^{1}$
}

\begin{abstract}
Our group at Bristol Robotics Laboratory has been working on a new robotic system for fracture surgery that has been previously reported [1]. The robotic system is being developed for distal femur fractures and features a robot that manipulates the small fracture fragments through small percutaneous incisions and a robot that re-aligns the long bones. The robots controller design relies on accurate and bounded force and position parameters for which we require real surgical data. This paper reports preliminary findings of forces and torques applied during bone and soft tissue manipulation in typical orthopaedic surgery procedures. Using customised orthopaedic surgical tools we have collected data from a range of orthopaedic surgical procedures at Bristol Royal Infirmary, UK. Maximum forces and torques encountered during fracture manipulation which involved proximal femur and soft tissue distraction around it and reduction of neck of femur fractures have been recorded and further analysed in conjunction with accompanying image recordings. Using this data we are establishing a set of technical requirements for creating safe and dynamically stable minimally invasive robot-assisted fracture surgery (RAFS) systems.
\end{abstract}

\section{INTRODUCTION}

There is an increased interest in the medical community for minimally invasive surgery (MIS), in which the surgeon manipulates instruments inserted into the patient through small incisions. There are benefits both for the patient's outcome (lower risk of infection, less soft tissue damage, less blood lose) as well as for the health systems supporting the operation (shorter hospital stays and lower re-hospitalisation risks due to complications).

In MIS, the surgeon has no direct contact with internal body parts thus haptic and tactile feedback in this interaction is minimum or non-existent. This feedback is crucial for better performing the procedure in question and a growing body of research is focusing on providing the surgeons with this information via some form of force/torque sensing method. Most studies have so far focused on MIS for pelvic surgery where force-feedback is added to laparoscopic instruments using various sensing technologies [2], [3], [4].

In minimally invasive fracture surgery, the issue of forcefeedback for the lower limb fractures relates to the manual manipulation and reduction of bone fragments in two distinctive ways. Firstly, the small fragments are manipulated

\footnotetext{
*This work was funded by the National Institute for Health Research (NIHR)'s Invention for Innovation (i4i) Programme, UK.

${ }^{1}$ Ioannis Georgilas, Giulio Dagnino and Sanja Dogramadzi are with Bristol Robotics Laboratory, University of the West of England, Coldharbour Lane, BS16 1QY, Bristol, United Kingdom ioannis.georgilas@uwe.ac.uk

${ }^{2}$ Payam Tarassoli and Roger Atkins are with the Bristol Royal Infirmary, Upper Maudlin Street, BS2 8HW, Bristol, United Kingdom
}

through externally drilled pins, and secondly, re-alignment of the limb is carried out by manipulating the tibia in respect to the femur. The overall goal is the use of a robotic system [1] to perform surgical procedures that involve moving individual fragments to the correct anatomical position, using a high precision parallel-robot [5], while also creating enough manipulation space, by applying traction to the affected limb using an external robotic device. Hence for the robotic system to be appropriately designed and safeguarded the forces and torques used for both the bigger and smaller fragment manipulation must be known and well understood. The current state of our study follows from our previous report on the force-torque measurement system for fracture surgery [6].

The related work in this field includes developments of surgical robots for fracture reduction of femur. Some of these robots e.g. [7], [8] are equipped with the force measuring sensors to compensate for the inaccuracy of bone motion caused by surrounding ligaments and muscles. Forces and torques have been measured and reported in phantoms [7] and in patients during femur fracture reduction [8]. The force-feedback is also crucial for the fracture reduction path planning since areas of high forces indicate soft tissue obstructions and should be avoided [9].

In the current preliminary study, we are presenting results from 8 fracture surgical operations. The data show measurements we acquired in surgical operations where surgeons displace small bone fragments, or larger bone structures. Specifically, the first set of measurements (4 operations) was gathered with an instrumented periosteal elevator [6], while the second set (4 operations) with an instrumented traction table.

The structure of this paper is as follows; in Section III we are giving the description of the hardware and software systems we used to collect the data, as well as the description of the surgical procedures we recorded and the analysis approach we utilised. In Section III] we present some indicative data from the studies that are giving the most relevant results. In Section [V], we are analysing the data collected in order to establish the technical requirements for the robot-assisted system, the internal parallel-robot and the external robotic mechanism.

\section{MATERIAL AND METHODS}

\section{A. Hardware and Software Description}

As our force-torque sensor for the instrumentation of the periosteal elevator and the traction table we are using a 6DOF load-cell (FTSens, Istituto Italiano di Tecnologia, Italy), 
Fig. 1(a) to simultaneously measure forces and torques applied to bone fragments by the surgeon in-vivo. The technical characteristics of the FTSens are, maximum force of $\pm 2000 \mathrm{~N}$ and $\pm 1500 \mathrm{~N}$ for $\mathrm{z}$-axis, and $\mathrm{xy}$-axes respectively, and resolution of $0.25 \mathrm{~N}$ for all three. Regarding torques, $\pm 25 \mathrm{Nm}$ and $\pm 35 \mathrm{Nm}$ around $\mathrm{z}$-axis, and xy-axes, with a resolution of $0.004 \mathrm{Nm}$ and $0.005 \mathrm{Nm}$ respectively. The data is transmitted via CAN-bus, a standard industrial-level transmission bus.

The periosteal elevator is a common tool in orthopaedic surgery used to handle soft tissues and bone fragments during fracture surgery. For the current study it has been cut in two parts between the tip and the handle with the FTSens been fixed in between. The tip of the device has been kept intact in order to assure bio-compatibility, but it can be removed from the device to allow proper sterilisation according to the hospital procedures. A $5 \mathrm{~mm}$ Acetal disk is separating the tip and the FTSens, Fig. 1(b) Six plastic PEEK socket head screws fasten the tip to the FTSens to ensure nonconductivity for patient safety. A formal verification process has been conducted for checking the insulation parameters according to clause 4.10 of IEC60601-1 given that the device is classified as a Safety Extra-Low Voltage (SELV). During the measurements, a protocol has been followed: the tip of the device was sterilised in autoclave and the other parts of the device (FTSens and device handle) were put inside a sterile endoscope drape, leaving only the sterile tip in contact with the tissues, Fig. 1(c) Given the change in size and weight, before each operation the surgeon was asked to confirm if he/she was comfortable to proceed with using the tool. In all of the cases the surgeons considered the alteration minimal and of no effect to their ability to operate.

The traction table is a structure added onto an operating table that allows application of traction and rotation to the limb, usually leg. Its adaptation was less radical, since the traction table is a modular structure that can be assembled as required just before the operation. As a result we created an insert that contains the FTSens, Fig. 1(d) and can be coupled with the existing structure of the traction table, Fig. 1(e). Since the traction table is not normally sterilised but covert with a surgical drape, no special sterilisation measures had to be taken. The length of the data cable was selected to ensure that the measuring equipment was away from the operating surgeon's working space. Since the insert with the load cell is in a direct contact with the patient, and the insulating material (foot rest and straps) is used to insulate the patient's foot, no special measures regarding conductivity had to be used.

In order to simplify data analysis we acquire force/torque measured by the FTSens together with visual information of the operation, following the tip in the periosteal elevator operation,and overall surgery views for the traction table, while recording surgeon's voice describing the manoeuvres. All recordings have been integrated into a Force Measurement Software (FMS) developed in LabView and running on a laptop PC. A graphical user interface (GUI) [6] allows the user to acquire and visualise the force/torque data in real-time together with video and audio information provided

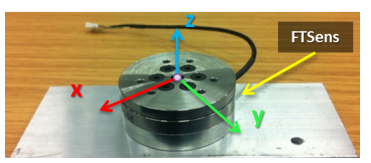

(a)

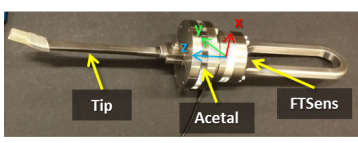

(b)

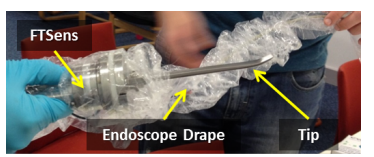

(c)

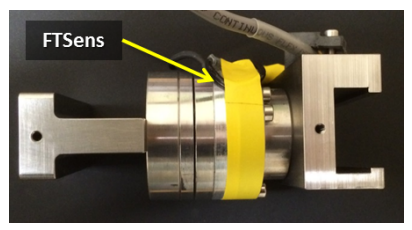

(d)

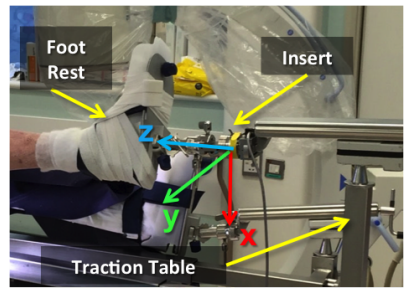

(e)
Fig. 1. Hardware used in the study, (a) The FTSens load-cell, (b) the instrumented periosteal elevator, (c) the sterilised setup for the elevator, (d) the traction table insert, (e) the full traction table assembly.

by a USB camera with a microphone. These three sources of information are time synchronised and simultaneously recorded for off-line analysis.

\section{B. Surgical Operations}

The selected surgical procedures were most relevant for understanding forces being applied during soft tissue and fragment manipulation and long bones alignment. The recruited patients cover a spectrum of sex, age and weight providing more complete results. All the patients gave informed consent and all the necessary measures to ensure privacy protection and patient safety were implemented.

For the four adapted periosteal elevator procedures we are presenting here, a variety of soft and bonny tissue interactions were measured. Specifically manipulation of muscle tissue in the coxal region, manipulation of fibrous tissue in the tarsal region, removal of bone scar tissue and manipulation of fragments in the crural region, and finally manipulation of periosteum in the crural region. The removal procedure is performed by repetitive movements along the axis of the tool (Z), with small diagonal movements (X, Y).

The four procedures where the instrumented traction table was used and are presented here were all extra-articular proximal femur fractures, i.e. coxal region. The traction is performed by adjusting the position and rotation of the traction table, Fig. 1(e), in respect to the fixed point of the patient's leg. This is performed in small steps, since the operating surgeon has to evaluate the positions of the fragments using intra-operative fluoroscopic imaging between each force/torque increment. Table I summarises the operations, the tissue or fracture type with the region operated, as well as the patient information.

\section{Data Analysis Methods}

A great spectrum of analysis methods could be used on the data collected during this study. In this preliminary report we are going to focus only on data that contributes the most 
TABLE I

Procedures MEASURED

\begin{tabular}{|c|c|c|}
\hline $\begin{array}{c}\text { Test } \\
\text { Code }\end{array}$ & $\begin{array}{c}\text { Tissue (Body Region) or } \\
\text { Fracture Type (AO classification) }\end{array}$ & $\begin{array}{c}\text { Patient Info } \\
\text { (S/Age/Weight) }\end{array}$ \\
\hline \hline PeE01 & Muscle tissue (Pelvic) & $\mathrm{M} / 50 / 80$ \\
\hline PeE02 & Fibrous tissue (Tarsal) & $\mathrm{M} / 50 / 80$ \\
\hline PeE03 & Bone scar tissue and fragments (Crural) & $\mathrm{M} / 25 / 75$ \\
\hline PeE04 & Periosteum (Crural) & $\mathrm{M} / 25 / 75$ \\
\hline TrT01 & Pertrochanteric Simple (31-A1) & $\mathrm{F} / 55 / 60$ \\
\hline TrT02 & Pertrochanteric Multifragmentary (31-A2) & $\mathrm{F} / 75 / 50$ \\
\hline TrT03 & Subcapital with no displacement (31-B1) & $\mathrm{F} / 83 / 50$ \\
\hline TrT04 & Subcapital with displacement (31-B1) & $\mathrm{M} / 53 / 110$ \\
\hline
\end{tabular}

to the objectives of the research, i.e. the design parameters regarding force and torques required to be exerted by a medical orthopaedic robot. For this reason we are going to report maximum forces, average values, and duration of application.

\section{RESULTS}

\section{A. Periosteal Elevator Measurements}

In Fig. 2 an indicative experiment of the periosteal elevator measurements, operation PeE03, is being presented, a link to the graphs for the other three operations can be found in the Appendix The nature of the manipulations are primarily to remove tissue from the bone, except from marked zone A in Fig. 2, where the displacement of incorrectly healed bone were performed before the removal of scar tissue from the surface of the bone, marked zone B in Fig. 2 Regarding the magnitude of the forces and torques exerted, the maximum observed force was $96.95 \mathrm{~N}$ along the Z-axis, with $\mathrm{X}$, $\mathrm{Y}$-axis forces maxing at $23.39 \mathrm{~N}$. The maximum torque was $4.17 \mathrm{Nm}$ around $\mathrm{Y}$-axis with torques around $\mathrm{X}, \mathrm{Z}$-axis maxing in average at $1.89 \mathrm{Nm}$. Table $\Pi$ II shows the maximum and average maximum values for the measured forces and torques. Duration of forces/torques applied depends on the surgeon's individual technique, and ranges from $0.1 \mathrm{sec}$ to $2.3 \mathrm{sec}$ (indicative regions can be seen in the graphs following the link in the Appendix).

\section{TABLE II}

MAXimum Force/Torque Values For Periosteal EleVator EXPERIMENTS

\begin{tabular}{|c||c|c|c|c|c|c|}
\hline & $\begin{array}{c}\text { FX } \\
(\mathrm{N})\end{array}$ & $\begin{array}{c}\text { FY } \\
(\mathrm{N})\end{array}$ & $\begin{array}{c}\text { FZ } \\
(\mathrm{N})\end{array}$ & $\begin{array}{c}\text { TX } \\
(\mathrm{Nm})\end{array}$ & $\begin{array}{c}\text { TY } \\
(\mathrm{Nm})\end{array}$ & $\begin{array}{c}\text { TZ } \\
(\mathrm{Nm})\end{array}$ \\
\hline \hline PeE01 & $\underline{23.39}$ & 15.54 & 75.49 & 1.32 & 3.75 & 0.12 \\
\hline PeE02 & 15.31 & 19.59 & $\mathbf{9 6 . 9 4}$ & 1.56 & $\mathbf{4 . 1 7}$ & 0.39 \\
\hline PeE03 & 20.94 & 12.06 & 80.82 & $\underline{1.89}$ & 3.23 & 1.37 \\
\hline PeE04 & 19.73 & 14.36 & 45.48 & 0.49 & 0.87 & 0.18 \\
\hline \hline AVG MAX & 19.84 & 15.39 & 74.68 & 1.31 & 3.00 & 0.52 \\
\hline
\end{tabular}

\section{B. Traction Table Measurements}

An indicative traction table measurement is shown in Fig. 3, operation TrT02, a link to the graphs for the other three operations can be found in the Appendix.

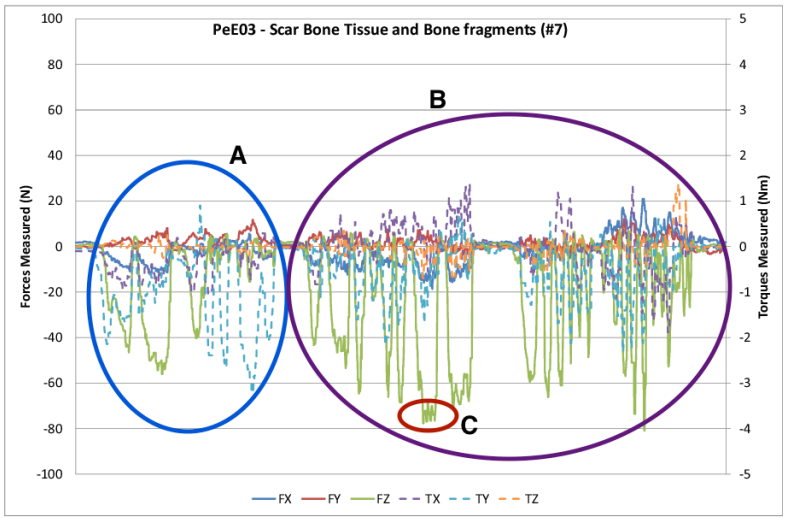

Fig. 2. Indicative forces/torques diagrams for the periosteal elevator experiments. Graphs are using two vertical axes. Left axis is Force measured in $\mathrm{N}$, right axis is Torque measured in Nm. Continuous lines are forces, blue (along $\mathrm{X}$ ), red (along $\mathrm{Y}$ ), and green (along $\mathrm{Z}$ ). Dashed lines are torques, purple (around $\mathrm{X}$ ), turquoise (around $\mathrm{Y}$ ), and orange (around $\mathrm{Z}$ ). Marked zones A and B indicate different parts of the operation. Marked zone C indicate duration of application of forces/torques. Graphs for other experiments can be seen in Appendix.

As anticipated, the forces/torques recorded are of higher value than with the periosteal elevator due to the nature of the procedure and the amount of soft tissue involved. The maximum recorded force is $604.53 \mathrm{~N}$ along $\mathrm{Z}$-axis , with $\mathrm{X}, \mathrm{Y}$-axis average maxing at $64.18 \mathrm{~N}$. Regarding torques applied, the maximum recorded is $13.33 \mathrm{Nm}$ around the $\mathrm{Y}$ axis with X,Z-axis average maxing at $9 \mathrm{Nm}$. Table III shows the maximum and average maximum values for the measured forces and torques. Duration of force/torque is not relevant to this procedures, since traction is applied and retained until the fracture gets fixated.

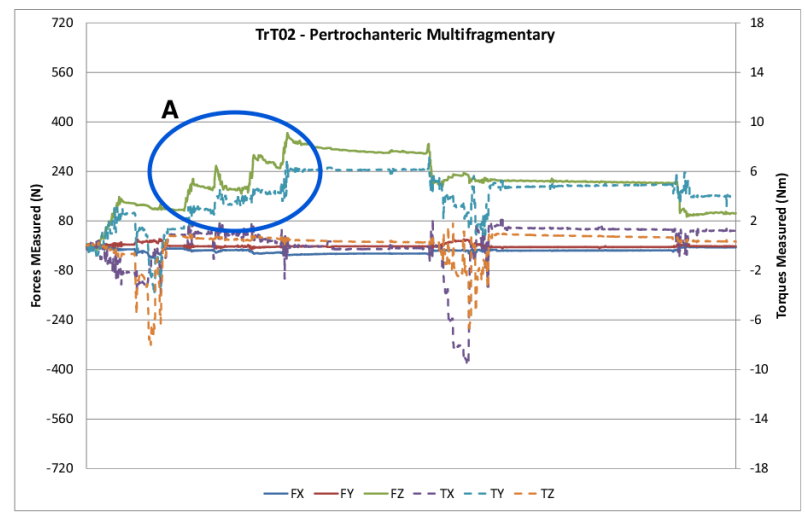

Fig. 3. Indicative forces/torques diagrams for the traction table experiments Graphs are using two vertical axes. Left axis is Force measured in N, right axis is Torque measured in $\mathrm{Nm}$. Continuous lines are forces, blue (along $\mathrm{X}$ ), red (along $\mathrm{Y}$ ), and green (along Z). Dashed lines are torques, purple (around $\mathrm{X}$ ), turquoise (around $\mathrm{Y}$ ), and orange (around $\mathrm{Z}$ ). Zone A indicate the stepped application of the traction forces. Graphs for other experiments can be seen in Appendix.

\section{DISCUSSION}

The data collected for the periosteal elevator procedure confirms the method used for tissue manipulation being 
TABLE III

MAXimum Force/Torque VALUES FOR Traction TABle

EXPERIMENTS

\begin{tabular}{|c||c|c|c|c|c|c|}
\hline & $\begin{array}{c}\text { FX } \\
(\mathrm{N})\end{array}$ & $\begin{array}{c}\text { FY } \\
(\mathrm{N})\end{array}$ & $\begin{array}{c}\text { FZ } \\
(\mathrm{N})\end{array}$ & $\begin{array}{c}\text { TX } \\
(\mathrm{Nm})\end{array}$ & $\begin{array}{c}\text { TY } \\
(\mathrm{Nm})\end{array}$ & $\begin{array}{c}\text { TZ } \\
(\mathrm{Nm})\end{array}$ \\
\hline \hline TrT01 & 49.03 & 32.17 & $\mathbf{6 0 4 . 5 3}$ & 9.49 & $\mathbf{1 3 . 3 3}$ & 5.74 \\
\hline TrT02 & 38.98 & 21.82 & 364.61 & 9.56 & 7.16 & 7.99 \\
\hline TrT03 & $\underline{64.18}$ & 54.85 & 160.33 & 8.69 & 11.83 & 4.09 \\
\hline TrT04 & 38.22 & 39.48 & 174.85 & 6.68 & 6.12 & 4.95 \\
\hline \hline AVG MAX & 47.61 & 37.09 & 326.09 & 8.61 & 9.61 & 5.70 \\
\hline
\end{tabular}

repetitive movements along the axis of the tool with small diagonal movements (figures can be found via the link in the Appendix). The highest forces measured along Z-axis are due to the surgeon applying high forces to penetrate the tissue with the sharp tip of the periosteal elevator. Since our purpose is manipulation of fragments in the body, the maximum forces recorded along $\mathrm{X}$ and $\mathrm{Y}$ axes are more relevant, hence the maximum of $24 \mathrm{~N}$ will be set as a technical requirement for the parallel-robot. The torques measured are difficult to identify if are related to tissue or bone manipulation. But given that the procedure of tissue removal requires high "lifting" torques i.e. around Y-axis, we can assume that torques around $\mathrm{X}$ and $\mathrm{Z}$ axes are more suitable for the technical requirement, and thus the value of $2 \mathrm{Nm}$ must be met.

Regarding the traction table experiments, it should be clarified that the first two operations required significantly more force/torque than the other two due to the type of fracture, involving the collapse of the femur and rotation of the neck-and-head fragment. None the less, the same method of adjusting the traction in steps was used and can be seen in marked zones A of all operations, (Figs 3). The most significant force value for our study is the highest recorded, i.e. along Z-axis, hence the external robotic system should meet the requirement of $650 \mathrm{~N}$ of traction but should also be able to apply at least $65 \mathrm{~N}$ along $\mathrm{X}$ and $\mathrm{Y}$ axes. For the torques required, all values recorded are at the same range; hence the requirement for the external robotic system should be set at $15 \mathrm{Nm}$ around all axes.

\section{CONCLUSIONS}

In this work we presented the experimental setup, data collected and preliminary analysis of forces applied during soft tissue and bone manipulation in typical orthopaedic surgery. Our primary goal was to establish a set of initial technical requirements for a new generation of minimally invasive robot-assisted fracture surgery (RAFS) systems. Such system will help the surgeon to operate optimally while protecting the patient from open-surgery dangers.

The requirements reported here will be used to design and implement a force controller and optimise the robotic platforms(i.e. parallel-robot and external robotic mechanism) for the RAFS system developed in the BRL, but also the data reported will benefit the research and clinical community with an indication of the dynamic requirements of fracture surgery. This knowledge can be used to create a database for surgical skills evaluations allowing objective measurement of skill, improving movement efficiency, and reducing softtissue injuries [10]. Future development will be reporting on more study measurements and an extensive analysis of the data collected.

\section{APPENDIX}

The figures for the other 3 periosteal elevator and 3 traction table experiments can be found in Medical Robotics theme page at BRL website.

\section{ACKNOWLEDGMENT}

This study received approval by the National Research Ethics Committee, with reference number 14/WM/0085.

This is a summary of independent research funded by the National Institute for Health Research (NIHR)'s Invention for Innovation (i4i) Programme. The views expressed are those of the author(s) and not necessarily those of the NHS, the NIHR or the Department of Health.

\section{REFERENCES}

[1] D. Raabe, S. Dogramadzi, and R. Atkins, "Semi-automatic percutaneous reduction of intra-articular joint fractures - An initial analysis," in 2012 IEEE International Conference on Robotics and Automation (ICRA), pp. 2679-2684, May 2012.

[2] A. Menciassi, A. Eisinberg, M. C. Carrozza, and P. Dario, "Force sensing microinstrument for measuring tissue properties and pulse in microsurgery," IEEE/ASME Transactions on Mechatronics, vol. 8, no. 1, pp. 10-17, 2003.

[3] H. Song, H. Kim, J. Jeong, and J. Lee, "Development of FBG sensor system for force-feedback in minimally invasive robotic surgery," in Proceedings of the International Conference on Sensing Technology, ICST, pp. 16-20, 2011.

[4] J. B. Gafford, S. Member, S. B. Kesner, A. Degirmenci, R. J. Wood, R. D. Howe, and C. J. Walsh, "A Monolithic Approach to Fabricating Low-Cost , Millimeter-Scale Multi-Axis Force Sensors for MinimallyInvasive Surgery," in 2014 IEEE International Conference on Robotics and Automation (ICRA), pp. 1419-1425, 2014.

[5] I. Georgilas, G. Dagnino, P. Tarassoli, R. Atkins, and S. Dogramadzi, "Robot-assisted system for joint fracture surgery," in 2015 Computer Assisted Orthopaedic Surgery (CAOS) International Meeting, 2015. Accepted.

[6] G. Dagnino, I. Georgilas, P. Tarassoli, R. Atkins, and S. Dogramadzi, "Force-torque measurement system for fracture surgery," in $2015 \mathrm{Com}$ puter Assisted Orthopaedic Surgery (CAOS) International Meeting, 2015. Accepted.

[7] T. Douke, Y. Nakajima, Y. Mori, S. Onogi, N. Sugita, M. Mitsuishi, M. Bessho, S. Ohhashi, K. Tobita, I. Ohnishi, I. Sakuma, T. Dohi, Y. Maeda, T. Koyama, N. Sugano, K. Yonenobu, Y. Matsumoto, and K. Nakamura, "Control of fracture reduction robot using force/torque measurement.," in 30th Annual International Conference of the IEEE Engineering in Medicine and Biology Society, EMBC, pp. 3265-3268, 2008.

[8] T. Gösling, R. Westphal, J. Faülstich, K. Sommer, F. Wahl, C. Krettek, and T. Hufner, "Forces and torques during fracture reduction: Intraoperative measurements in the femur," Journal of Orthopaedic Research, vol. 24, no. 3, pp. 333-338, 2006.

[9] J. Buschbaum, R. Fremd, T. Pohlemann, and A. Kristen, "Computerassisted fracture reduction: a new approach for repositioning femoral fractures and planning reduction paths.," International journal of computer assisted radiology and surgery, vol. 10, pp. 149-59, Feb. 2015.

[10] J. Rosen, M. MacFarlane, C. Richards, B. Hannaford, and M. Sinanan, "Surgeon-tool force/torque signatures-evaluation of surgical skills in minimally invasive surgery.," Studies in health technology and informatics, vol. 62, pp. 290-296, 1999. 